\title{
Influence of electrical stimulation of the tibialis anterior muscle in paraplegic subjects. 2. Morphological and histochemical properties
}

\author{
L Rochester ${ }^{1}$, MJ Barron ${ }^{2}$, CS Chandler ${ }^{1}$, RA Sutton ${ }^{4}$, S Miller $^{3}$ and MA Johnson ${ }^{2}$ \\ ${ }^{1}$ Institute of Health Sciences, University of Northumbria at Newcastle, NE7 7XA; ${ }^{2}$ Division of Neurobiology and \\ ${ }^{3}$ Department of Child Health, Newcastle upon Tyne University, NE2 $4 \mathrm{HH} ;{ }^{4}$ Regional Spinal Injuries Unit, \\ Hexham General Hospital, NE46 1QJ, UK
}

\begin{abstract}
In adult paraplegic subjects one tibialis anterior muscle received daily electrical stimulation for 4 weeks at twice the motor threshold to determine changes of morphological and histochemical profiles (this paper) and of contractile properties (preceding paper). ${ }^{1}$ Bilateral biopsies, obtained 4 weeks before, and immediately after, electrical stimulation, were studied for fibre type proportions, fibre diameters, oxidative capacity, microvasculature and histopathology. Before stimulation the biopsies showed disuse with increased type 2 fibre proportions and decreased oxidative capacity (succinate dehydrogenase (SDH) activity). The effects of two stimulus patterns ${ }^{1}$ were compared. Following stimulation SDH activity increased significantly in all stimulated muscles. Inconsistent changes occurred in fibre type proportions, fibre diameters, capillary density and capillary/fibre ratios. Both stimulus patterns evoked similar results. In five/seven subjects subsarcolemmal vacuolation was observed. Electrical stimulation for 4 weeks at only twice motor threshold improves oxidative capacity, but different stimulus parameters are probably needed for significant fibre type conversion.
\end{abstract}

Keywords: skeletal muscle; morphology; histochemistry; electrical stimulation; paraplegic subjects

\section{Introduction}

The activity of motoneurons determines the properties of the muscle fibres they supply ${ }^{2,3}$ and disuse, as a result of neurological or orthopaedic trauma, may have profound effects. ${ }^{4-8}$ These reports demonstrate that in the extreme case of prolonged disuse following spinal cord injury the proportion of type 2 (fast twitch) fibres increases, muscle fibre atrophy occurs and the oxidative capacity of the muscle is much reduced.

Electrical stimulation of muscle for prolonged periods has been shown to reverse or help to prevent the effects of disuse ${ }^{9-11}$ and to increase capillary density. ${ }^{12}$ In animal models this type of stimulation has been shown to alter morphological and histochemical properties. ${ }^{13}$ The aim of the present paper was to determine the extent to which these properties might be altered in human subjects with prolonged muscular disuse and how far electrical stimulation might reverse the changes. It was also of importance to know how far these changes might correlate with the changes of contractile properties (Rochester $e t a l) .{ }^{1}$

The level of succinate dehydrogenase (SDH) activity in skeletal muscle is known to correlate with fatigueresistance. ${ }^{14}$ The stimulus regimes used in this study might be expected to enhance fatigue resistance and

Correspondence: S Miller it was therefore appropriate to monitor the muscle biopsies for evidence of altered SDH activity. It was also considered possible that individual muscle fibre types might respond differently to stimulation. Microphotometric enzyme assay enabled SDH activity to be estimated in single muscle fibres and in populations of histochemically defined fibre types and was thus ideal for the detection of any differential response.

In the majority of studies in human subjects electrical stimulation has been applied to relatively normal healthy muscles. It is not known if disused muscles can withstand the levels of current over prolonged periods of time without suffering damage - an important factor in the therapeutic application of the technique. For these considerations the current pulses used in the present study were set at only twice the motor threshold.

A brief report of the morphological and histochemical results has been published. ${ }^{15}$

\section{Materials and methods}

\section{Subjects}

A group of seven paraplegic subjects aged 19-43 years took part in the study; details of these subjects are given in the preceding paper. ${ }^{1}$ Informed written consent was obtained from each subject and the study was 
approved by the regional health authority ethical committee.

\section{Electrical stimulation}

The techniques used are described in the preceding paper $^{1}$ and are only summarised here. The tibialis anterior muscle was stimulated percutaneously with electrodes placed over the muscle belly. The strength of current used was twice the threshold for obtaining muscle contraction. Two stimulus patterns were used broadly similar to those described by Kernell et al ${ }^{12}$ each pattern was administered for a 4 week period. (a) Slow pattern: $10 \mathrm{~Hz}$ stimulus bursts lasting $5.5 \mathrm{~s}$ with pauses of $5.5 \mathrm{~s}$, given in three periods of $48 \mathrm{~min}$ daily; this amounts to an overall stimulation time of $5 \%$ daily time. (b) Slow/fast pattern: slow $(10 \mathrm{~Hz})$ pattern with added $100 \mathrm{~Hz}$ bursts comprising an extra $0.5 \%$ daily stimulation time. Three subjects received the slow stimulus pattern and four subjects the slow/fast pattern. Tibialis anterior muscles were stimulated on one side only.

\section{Muscle biopsies}

Biopsies from both the left and right tibialis anterior (TA) muscles were taken prior to treatment and after the period of electrical stimulation, using a conchotome procedure. The biopsies were obtained from a region of the muscle close to the sites of the two stimulating electrodes. The biopsy sites in the control leg and in the pre-stimulus condition of the stimulated leg were comparable. The muscle samples were transversely orientated and frozen using isopentane cooled to $-150^{\circ} \mathrm{C}$ in liquid nitrogen. Histological assessment was made using post-fixed frozen sections stained with haematoxylin and eosin.

\section{Fibre type proportions and fibre diameters}

Myofibrillar ATPase activity was demonstrated at $\mathrm{pH} 9.5$, after pre-incubatioin at $\mathrm{pH} 4.3$ or $\mathrm{pH} 4.6 .^{16}$ This allowed the differentiation of type 1 (slow-twitch oxidative), type $2 \mathrm{~A}$ (fast-twitch oxidative/glycolytic) and type $2 \mathrm{~B}$ (fast-twitch glycolytic fibres). Intermediate ' $2 \mathrm{C}$ ' fibres were also identified. Samples of 200 muscle fibres were used for measurements of muscle fibre diameters ${ }^{17}$ by means of a computer-aided system (Kontron Videoplan Kontron UK, Thame, UK). The prevalence of atrophy or hypertrophy was estimated using the method of Brooke and Engel ${ }^{18}$ for the calculation of atrophy factors (AF) and hypertrophy factors (HF).

\section{Oxidative capacity}

Succinate dehydrogenase (SDH) activity was used as an indicator of oxidative capacity and was measured in individual muscle fibres by microphotometric enzyme assay (MEA). Samples of 100 fibres per biopsy were used. The assay method is given in detail elsewhere. ${ }^{19}$ A Zeiss UMSP30 microphotometer Oberkochen, Germany, fitted with a computer-controlled scanning stage, was used to assay 25 muscle fibres simultaneously. Assay medium for SDH contained $130 \mathrm{mM}$ sodium succinate, $1.5 \mathrm{mM}$ nitro blue tetrazolium (NBT), $0.2 \mathrm{mM}$ phenazine methosulphate (PMS), $1.0 \mathrm{mM}$ sodium azide in $0.1 \mathrm{M}$ phosphate buffer, $\mathrm{pH}$ 7.0. The increase in absorbance $(\Delta \mathrm{A})$ due to the production of formazan has been shown to be linear, by regression analysis, for at least $10 \mathrm{~min}$, using $8 \mu \mathrm{m}$ sections of normal human muscle. A datahandling programme allowed the slope of the reaction in individual fibres (reaction rate) to be calculated. Muscle fibres which had been assayed were subject to retrospective fibre typing. This was done by comparing Polaroid photomicrographs of unreacted tissue sections, on which the assayed fibres were mapped, with the corresponding areas of serial section in which myofibrillar ATPase was demonstrated.

A series of six subjects from whom tibialis anterior muscle biopsies had been taken for routine diagnostic purposes, provided 'disease control' material for estimation of oxidative capacity. In four of these subjects there were no histopathological abnormalities. The clinical presentations in these subjects were, respectively: intermittent myalgia, myalgic encephalomyelitis, generalised muscle weakness, and possible recurrence of treated myositis (not substantiated by muscle biopsy). In the other two subjects there was mild electrophysiological and/or histochemical evidence of denervation and reinnervation. Although these control subjects cannot be considered unequivocally as normal controls, they provide an indication of the levels of $\mathrm{SDH}$ in the tibialis anterior muscles in subjects with no spinal cord lesion.

\section{Microvasculature}

Affinity cytochemistry (lectin binding) was used to localise the capillary network in muscle sections. Ulex europaeus agglutinin-1 (UEA-1) binds to fucose residues on vascular endothelial cells. ${ }^{20}$ UEA-1 was diluted 1:5000 in phosphate-buffered saline (PBS) containing $1 \%$ bovine serum albumin, $\mathrm{pH} 7.4$ and was applied to air-dried, acetone-fixed sections for $1 \mathrm{~h}$ at room temperature. After thorough washing $(\mathrm{PBS} \times 3$ over $30 \mathrm{~min})$ rabbit antibody to UEA-1 (1:6400) was applied for $1 \mathrm{~h}$ at room temperature. After thorough washing, alkaline phosphatase-conjugated swine anti-rabbit immunoglubulins (1:100) were applied for $30 \mathrm{~min}$ at room temperature (lectin and immunoreagents obtained from Dako Ltd, High Wycombe, UK). The alkaline phosphatase label was visualised using Naphthol ASTR phosphate as substrate, hexazotised New Fuchsin as coupling agent and incorporating levamisole as an inhibitor of endogenous alkaline phosphatase activity.

\section{Quantitation of capillary supply}

The same group of six 'disease control' patients was used to provide data on muscle microvasculature in subjects with intact spinal cord. Labelled capillaries were counted in four separate areas of tissue sections. 
Given an initial total sample area of $5 \mathrm{~mm}^{2}$, the area occupied by muscle fibres was generally $4.0-4.5 \mathrm{~mm}^{2}$. The proportion of the total sample area occupied by skeletal muscle fibres was estimated by means of a stereological (point-counting) technique. Blank areas, the area occupied by fat, fibrous connective tissue etc, were excluded, as were any non-transversely orientated areas of muscle. The total number of muscle fibres and the total number of capillaries within the sample area were counted. From these data, the capillary density (number of capillaries per $1 \mathrm{~mm}^{2}$ muscle fibre area) was calculated and also the average number of capillaries per muscle fibre (capillary/fibre ratio).

\section{Creatine kinase estimation}

In order to determine evidence of any muscle damage related to electrical stimulation, serum creatine kinase levels were measured before the stimulation period commenced and at weekly intervals until stimulation ceased. The level of this muscle enzyme was measured from samples of venous blood. These measurements were carried out only on the two last paraplegic subjects and were made after evidence of damage was observed histologically in some of the preceding paraplegic subjects tested.

\section{Data analysis}

The main test of statistical significance used was Student's $t$ test (2-tailed variant) which was applied in the analysis of the results of SDH assays, of capillary/ fibre ratios and of capillary densities. Analysis of abnormalities of the distributions of muscle fibre size was performed using the atrophy/hypertrophy factor system. ${ }^{18}$

\section{Results}

\section{Fibre type proportions}

The activity of myofibrillar ATPase after preincubation at $\mathrm{pH} 4.3$ and $\mathrm{pH} 4.6$ was assessed in a sample of 200 fibres per muscle biopsy, providing estimates of the proportions of types 1, 2A, 2B and 2C. The majority of the papaplegic subjects showed a marked decrease in the proportion of type 1 fibres compared with that of normal subjects (Figures 1,2), often falling outside the 95\% confidence limits. ${ }^{21}$ However, the magnitude of the decrease was not correlated with the length of time following spinal cord injury (see Table 1 in preceding paper). ${ }^{1}$ Subjects 2 and 3 both suffered spinal transection 7 years prior to biopsy, but, while subject 3 showed in all biopsies almost complete conversion to type 2 fibres with very few type 1 fibres, subject 2 showed preservation of type 1 fibres within the normal range.

In the biopsies following the 4 week period of electrical stimulation there were no clear trends in the proportions of the different fibre types. In some subjects, all the muscle biopsies showed fairly similar fibre type proportions, eg subjects 2, 3 and 6 (Figure 2). Subject 5, however, showed considerable variation between right and left biopsies even before electrical stimulation.
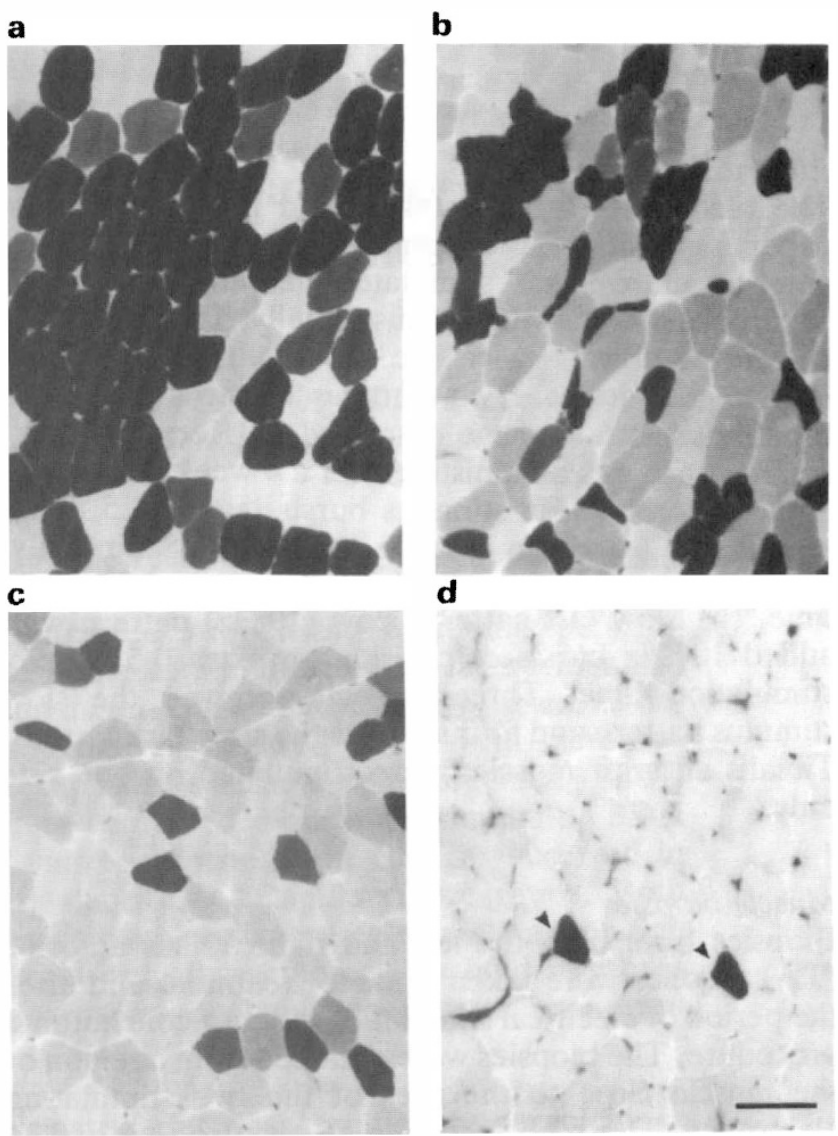

Figure 1 Fibre type proportions in the tibialis anterior muscle of paraplegic subjects. Myofibrillar ATPase after pre-incubation at $\mathrm{pH}$ 4.6. Type 1 fibres appear dark, type 2A fibres pale and type $2 \mathrm{~B}$ fibres intermediate. (a) Subject 7, showing a near-normal range of fibre diameters and preservation of a high proportion of type 1 fibres. (b) Subject 1 , showing selective atrophy of type 1 fibres and a moderate decrease in the proportion of type 1 fibres. (c) Subject 4 , showing a severe decrease in the proportion of type 1 fibres. (d) Subject 3, showing virtual absence of type 1 muscle fibres (arrows). Bar $=100 \mu \mathrm{m}$

Fibre type conversion is signalled by an increase in the proportion of type $2 \mathrm{C}$ (intermediate) fibres, which should not exceed $3 \%$ in normal skeletal muscle. ${ }^{22}$ Following electrical stimulation there were no consistent changes in the proportions of type $2 \mathrm{C}$ fibres. The proportions of type $2 \mathrm{C}$ fibres from the biopsies of stimulated and unstimulated legs were therefore pooled for each subject for comparison with the normal range. Subjects 2, 6 and 7 all showed mean type $2 \mathrm{C}$ proportions greater than $15 \%$. This might be expected in subjects 6 and 7 since the time following spinal transection was relatively short and the muscle fibre population would be likely to be in a state of conversion. Subjects 1,4 and 5 all had long-standing paraplegia with severely decreased type 1 proportions. In these subjects there was a smaller mean percentage of type $2 \mathrm{C}$ fibres $(5-10 \%)$, indicating less fibre type conversion at this stage. In subject 3 conversion to a type 2 fibre profile was virtually total with correspondingly few type $2 \mathrm{C}$ fibres. 
UNSTIMULATED $\quad$ STIMULATED

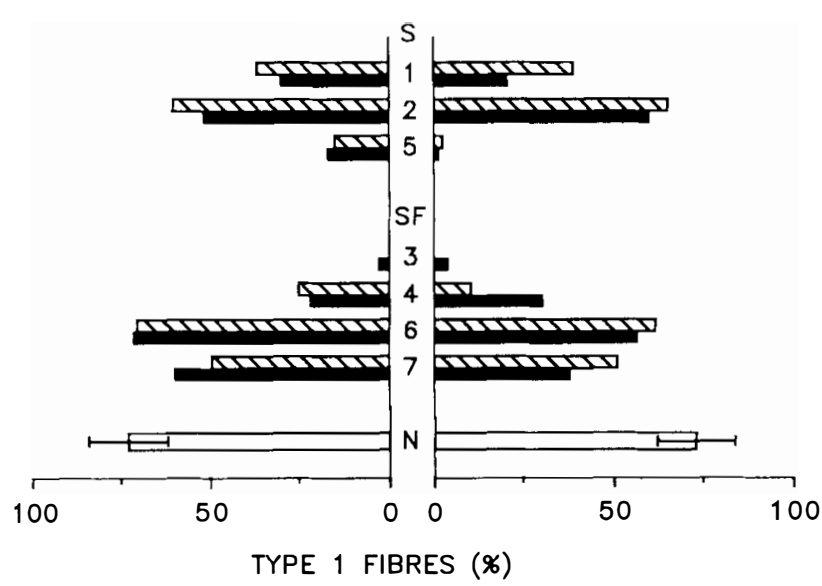

Figure 2 Proportions of type 1 fibres in stimulated and unstimulated limbs of paraplegic subjects before and after the stimulation period. Hatched and solid bars indicate biopsies 1 and 2 , respectively. The pattern of stimulation is shown above the subject groups $(\mathrm{S}=$ slow pattern; $\mathrm{S} / \mathrm{F}=$ slow/fast pattern). Subjects are numbered. Normal values are shown for comparison, open bar indicating mean \pm 2 standard deviations. (Note: in subject 3 biopsy 1 contained no type 1 fibres)

\section{Fibre diameters}

The mean and standard deviation of the diameters for each fibre type were determined from samples of 200 fibres in each biopsy (Figure 3a, b, c). It was found that whereas mean fibre diameters prior to electrical stimulation generally fell within the normal range, ${ }^{23}$ size distributions were often abnormal.

Atrophy and hypertrophy factors ${ }^{18}$ were calculated for each fibre type to determine if changes in size distribution had occurred before and after the period of electrical stimulation. Calculation of these factors takes into account the relative number of fibres which fall outside the normal range of diameters and also the degree of atrophy and hypertrophy shown by individual fibres.

Clear evidence of atrophy was observed in the majority of biopsies from paraplegic subjects, which may be associated with the physiological finding of decreased ability to generate torque. ${ }^{1}$ No evidence was obtained that electrical stimulation consistently reduces the incidence of atrophy. The incidence of increased atrophy factors and details of the involvement of individual fibre subpopulations (fibre types) are given in Table 1. Muscle fibre atrophy is, however, by no means a universal finding in these paraplegic subjects; three of the biopsies from subject 3 and two of the biopsies from subject 4 showed no evidence of atrophy of any muscle fibre type. Atrophy of type 1 fibres occurred in $20 / 28$ biopsies, type $2 \mathrm{~A}$ atrophy in $11 / 28$ biopsies and type $2 \mathrm{~B}$ atrophy in $5 / 28$ biopsies. In the $2 \mathrm{C}$ fibre populations atrophy was observed in $17 / 28$ biopsies. Discrepancies between successive biopsies of the same muscle are a further indication of the variability of these paraplegic muscles as a whole and the difficulty of avoiding sampling error in any biopsy procedure.

Muscle fibre hypertrophy occurred much more rarely than fibre atrophy. The most commonly involved fibre type was type $2 \mathrm{~B}$ ( $7 / 28$ biopsies). Type $2 \mathrm{~A}$ hypertrophy ( $2 / 28$ biopsies) and $2 \mathrm{C}$ fibre hypertrophy (1/28 biopsies) were rarely encountered and hypertrophy of type 1 fibres was not seen at all.

\section{Histopathological findings in paraplegic muscles}

The 28 muscle biopsies obtained showed a variety of histopathological features in addition to the abnormalities of fibre size and fibre type constitution already described (Table 2). The incidence of histopathological features varied widely between subjects. Acute muscle necrosis was a rare finding but fibre splitting and a 'whorled' appearance due to myofibrillar disorientation were common. Abnormalities of mitochondrial distribution leading to a 'floccular' or 'moth-eaten' appearance were frequently seen in succinate dehydrogenase preparations. In some biopsies increase in fibrous connective tissue, particularly in perimysial areas, was apparent and occasional biopsies showed some increase in interstitial fat.

Following the period of electrical stimulation mild subsarcolemmal vacuolation was observed in biopsies from the stimulated tibialis anterior muscle of $5 / 7$ subjects (Figure 4). It was possible that this feature

Table 1 Incidence of increased atrophy factors (AF) in individual fibre types of the tibialis anterior muscle in paraplegic subjects

\begin{tabular}{lllll}
\hline Subject & \multicolumn{2}{c}{ Unstimulated limb } & \multicolumn{2}{c}{ Stimulated limb } \\
\cline { 2 - 3 } & Biopsy 1 & Biopsy 2 & Biopsy 1 & Biopsy 2 \\
\hline 1 & $1,2 \mathrm{C}$ & $1,2 \mathrm{C}$ & $1,2 \mathrm{~A}, 2 \mathrm{C}$ & $1,2 \mathrm{C}$ \\
2 & $1,2 \mathrm{~A}, 2 \mathrm{C}$ & $1,2 \mathrm{C}$ & $1,2 \mathrm{~A}, 2 \mathrm{C}$ & $2 \mathrm{~B}$ \\
3 & - & - & - & 1 \\
4 & - & 1 & 1 & - \\
5 & $1,2 \mathrm{~A}, 2 \mathrm{C}$ & $1,2 \mathrm{~A}, 2 \mathrm{~B}, 2 \mathrm{C}$ & $1,2 \mathrm{~A}$ & $1,2 \mathrm{~A}, 2 \mathrm{~B}, 2 \mathrm{C}$ \\
6 & $1,2 \mathrm{~A}, 2 \mathrm{~B}, 2 \mathrm{C}$ & $1,2 \mathrm{~A}$ & $1,2 \mathrm{~A}, 2 \mathrm{~B}, 2 \mathrm{C}$ & $1,2 \mathrm{C}$ \\
7 & $2 \mathrm{C}$ & $1,2 \mathrm{C}$ & $1,2 \mathrm{~A} 2 \mathrm{C}$ & - \\
\hline
\end{tabular}

Fibre types are indicated by $1,2 \mathrm{~A}, 2 \mathrm{~B}$ and $2 \mathrm{C}$. No significant atrophy is indicated by - 

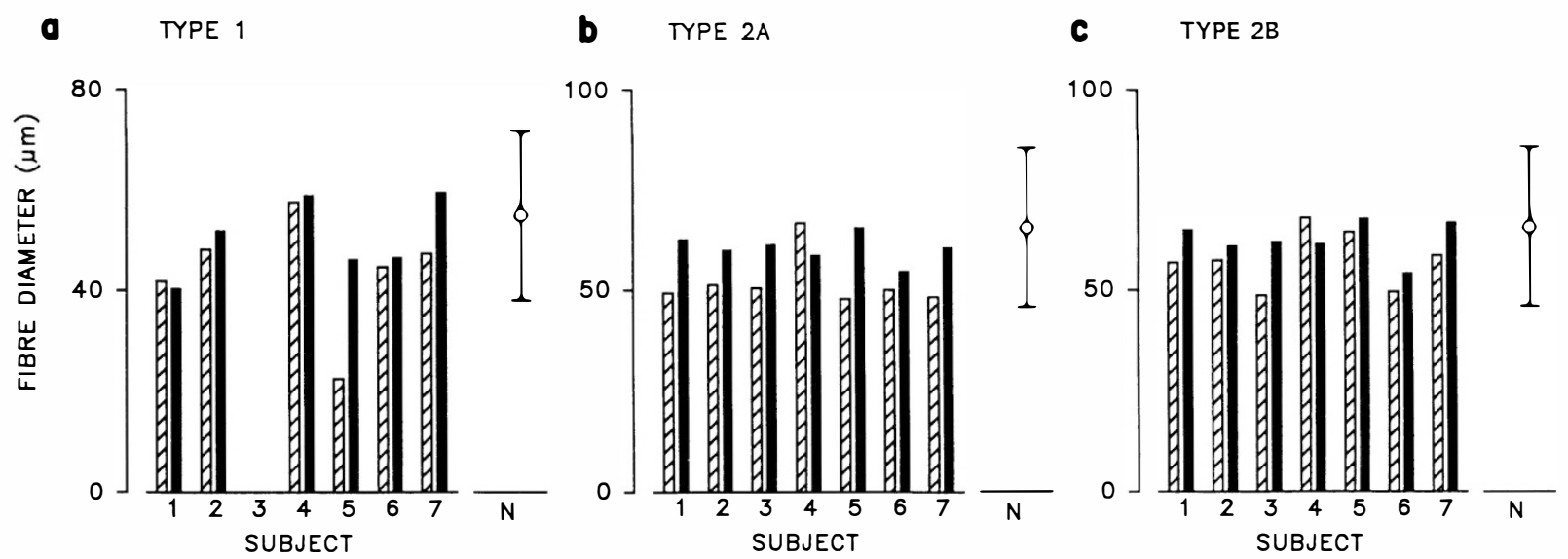

Figure 3 Mean fibre diameters in type 1 (a), type 2A (b) and type 2B (c) fibres, prior to the period of electrical stimulation. The normal range (mean and $95 \%$ confidence limits) is indicated by the open circle and error bars, and by $\mathrm{N}$ in the abscissa. Hatched and solid bars indicate stimulated and unstimulated limbs respectively

Table 2 Incidence of histopathological features in the tibialis anterior muscle of paraplegic subjects

\begin{tabular}{llllll}
\hline Subject & \multicolumn{2}{c}{ Unstimulated lamb } & & \multicolumn{2}{c}{ Stimulated limb } \\
\cline { 2 - 3 } \cline { 5 - 6 } & Biopsy & Biopsy & & Biopsy & Biopsy \\
& 1 & 2 & & 1 & 2 \\
\hline 1 & M & C, M, S & M & M, V \\
& M & M & M & C, M, S \\
3 & - & N & & - & M, N, V \\
4 & M & M & M, N, S & S, V \\
5 & N, S & M, S & S & C, S \\
6 & S & C, S & M, S & V \\
7 & - & S & - & V \\
\hline
\end{tabular}

$\mathrm{C}=$ connective tissue increase $\mathbf{M}=$ abnormal mitochondrial distribution; $\mathrm{N}=$ acute necrosis/phagocytosis; $\mathrm{S}=$ split or whorled fibres; $\mathrm{V}=$ subsarcolemmal vacuolation a

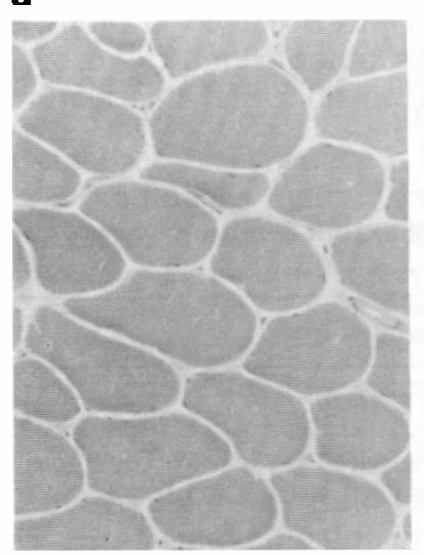

b

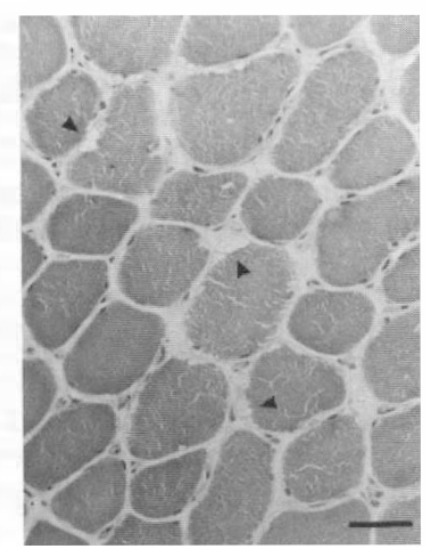

Figure 4 Subject 4 showing evidence of subsarcolemmal vacuolation after electrical stimulation (arrows). (a) Left tibialis anterior before the period of electrical stimulation. (b) Left tibialis anterior after the period of electrical stimulation. Haematoxylin and eosin; bar $=50 \mu \mathrm{m}$ might be an early indication of muscle fibre breakdown, but monitoring of serum creatine kinase levels in subjects 6 and 7 during electrical stimulation gave no indication of significant muscle fibre damage.

\section{Oxidative capacity}

In microphotometric enzyme assays of succinate dehydrogenase (SDH) activities were expressed as increase in absorbance $(\Delta \mathrm{A})$ per second. In the disease control group the fibre types showed a clear gradient of SDH activity with type 1 fibres having approximately $50 \%$ higher activity than type $2 \mathrm{~B}$ fibres, with the activity of type $2 \mathrm{~A}$ fibres intermediate between those of types 1 and $2 \mathrm{~B}$ (Figure 5a).

Prior to electrical stimulation the paraplegic subjects had reduced oxidative metabolic capacity (Figure 5b). Subjects 1 and 2 demonstrated values of SDH activity (pooled from all fibre types) below the mean of the control group, and subjects 3, 4 and 5 showed activity below the lower limit of the control group. Following electrical stimulation there was a significant increase in $\mathrm{SDH}$ activity in the stimulated legs of all subjects $(P<0.001$ in all cases $)$. Where the initial values of SDH activity were low the proportional increase following stimulation was large (Figure 5b, subjects 3, 4 and 5). The greatest increase in SDH activity (161\%) was observed in subject 4 , which exceeded the upper limit of activity in the disease control group. No differences were observed between the effects of the two stimulus patterns.

In the non-stimulated limbs, successive biopsies generally showed consistent values for SDH activity. For example the percentage differences between first and second biopsies in subjects $1,3,4,6$ and 7 were $+4 \%, 0 \%,-1 \%,+3 \%$ and $-11 \%$, respectively. However, in subjects 2 and 5 increases of $+25 \%$ and $+47 \%$ are difficult to interpret, since the successive muscle samples showed no gross differences in fibre type proportions or in the incidence of histopathological abnormalities. 
a

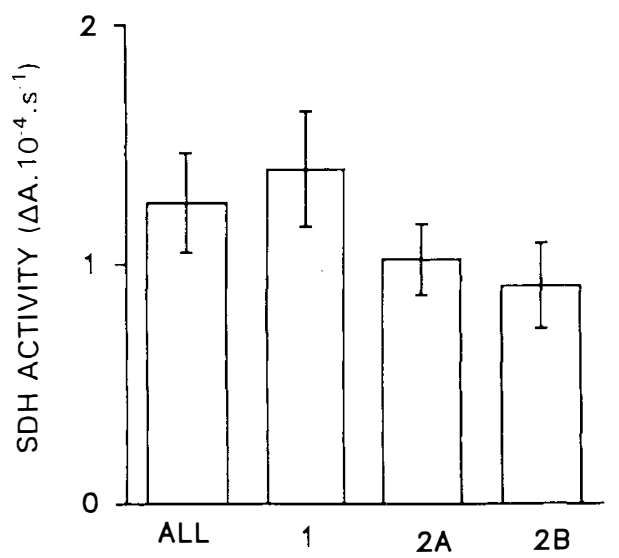

b

UNSTIMULATED

STIMULATED

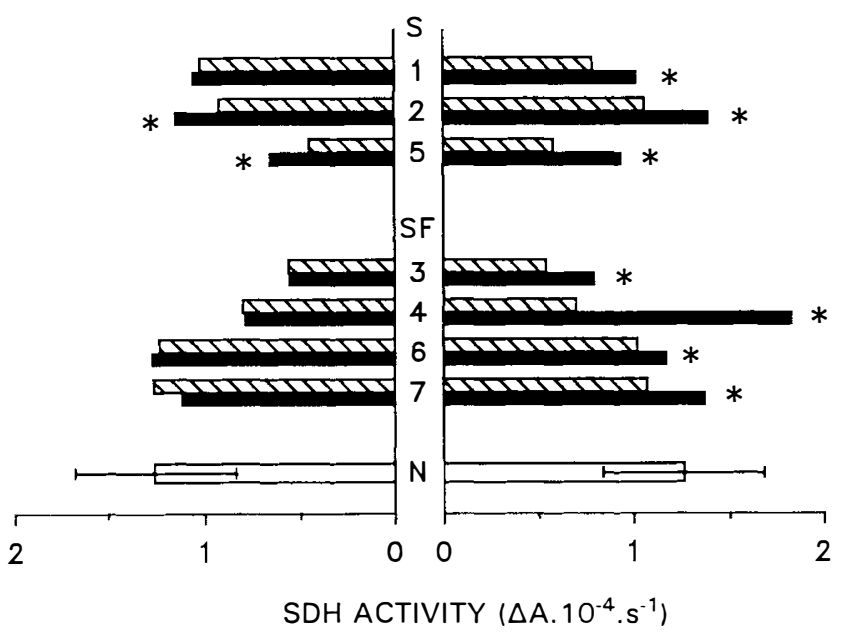

Figure 5 (a) Histogram showing succinate dehydrogenase activity in muscle fibre subpopulations in a group of six control subjects. Bars indicate \pm 1 standard deviation. (b) Succinate dehydrogenase activity in stimulated and unstimulated limbs of paraplegic subjects before and after the stimulation period. Hatched and solid bars indicate biopsies 1 and 2 , respectively. The pattern of stimulation is shown above the subject groups $(\mathrm{S}=$ slow pattern; $\mathrm{S} / \mathrm{F}=$ slow/fast pattern $)$. Subjects are numbered. The control group $(\mathrm{N})$ is shown for comparison, open bar indicating mean \pm 2 standard deviations. * significant increase $(P<0.001)$

\section{Microvasculature in paraplegic subjects}

The same group of disease control subjects were used to provide data on capillary/fibre ratio and capillary density. Capillary/fibre (C/F) ratio in this group of control patients was $1.12 \pm 0.31$, using sample areas of approximately $4.0 \mathrm{~mm}^{2}$. The range of values seen in this group is quite high (coefficient of variation $28 \%$ ) due to the presence of subjects with either an excess of small diameter fibres (low $\mathrm{C} / \mathrm{F}$ ratio) or of large diameter fibres (high $\mathrm{C} / \mathrm{F}$ ratio). Capillary density (number of capillaries per $\mathrm{mm}^{2}$ ) is independent of variation in fibre size and is generally less variable than $\mathrm{C} / \mathrm{F}$ ratios within any group of individuals. In the disease control group capillary density was $211 \pm 30$ per $\mathrm{mm}^{2}$ with a coefficient of variation of $14 \%$. The $95 \%$ confidence limits were $156-276$ for capillary density and $0.50-1.74$ for capillary/fibre ratio.

In the group of seven paraplegic subjects the capillary/fibre ratio was within the control range in all 28 biopsies, but the majority of values fell below the mean of the control group. When pre-stimul and post-stimul biopsies were compared, an overall trend towards a modest increase was observed (Figure 6), although the increase in subject $4(+62 \%)$ was the only instance where the difference was significant $(P<0.05)$. This subject also showed the greatest increase in SDH activity. Essentially the same results were obtained when capillary densities were compared, with only subject 4 showing a significant increase (Figure 7).

\section{Discussion}

The changes in the contractile properties of the tibialis anterior muscle in paraplegic subjects, reported in the

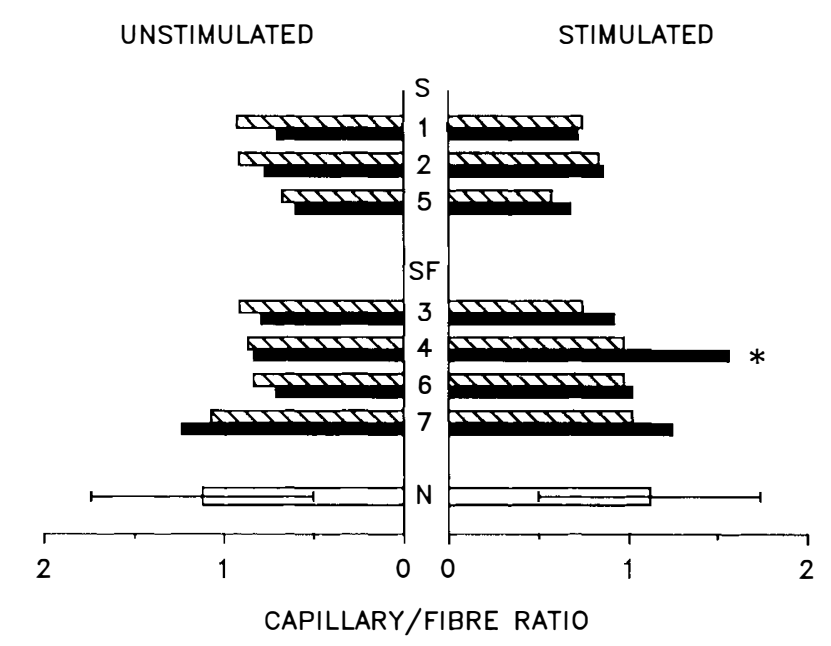

Figure 6 Capillary/fibre ratio in stimulated and unstimulated limbs of the paraplegic subjects, before and after the stimulation period. Hatched and solid bars indicate biopsies 1 and 2, respectively. The pattern of stimulation is shown above the subject groups $(\mathrm{S}=$ slow pattern; $\mathrm{S} / \mathrm{F}=$ slow $/$ fast pattern). Subjects are numbered. The control group is shown for comparison, open bar indicating mean \pm 2 standard deviations. *significant increase $(P<0.05)$

preceding paper ${ }^{1}$ following disuse and retraining with a 4 week period of electrical stimulation, have been largely supported by the present morphological and histochemical study of pre- and post-stimulation biopsies.

Effects of disuse following spinal cord transection Marked changes in the morphological and histochemical properties of the tibialis anterior were evident in 
a
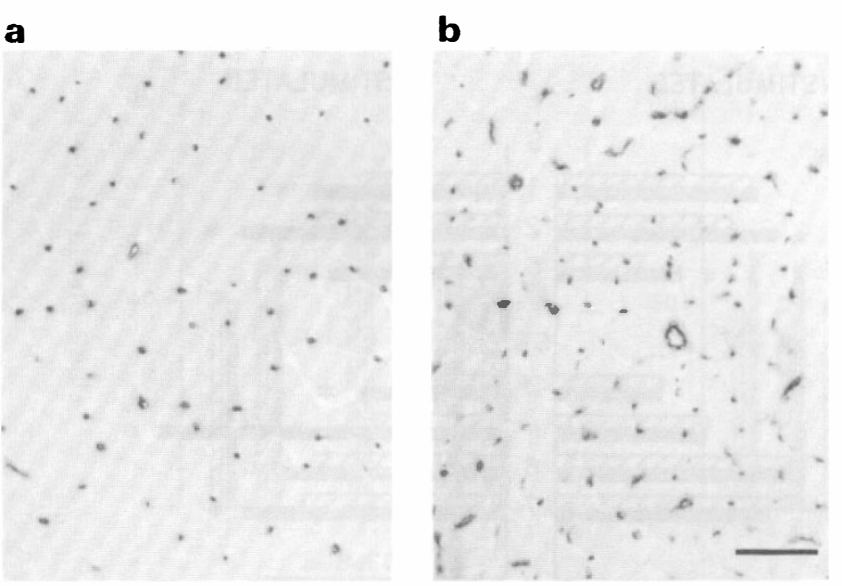

Figure 7 Distribution of capillaries in skeletal muscle before and after stimulation in subject 4. Capillaries are localised using Ulex europaeus agglutinin 1 (UEA-1) and alkaline phosphate labelling. (a) Capillary density in the tibialis anterior muscle before stimulation is lower than normal. (b) After stimulation, capillary density is increased to within the normal range. $\mathrm{Bar}=100 \mu \mathrm{m}$.

the paraplegic subjects who had suffered traumatic complete spinal transection 1-14 years previous to testing. They included an increase in the proportion of type 2 fibres, atrophy of muscle fibres, decreased activity of SDH in all fibre types and some evidence of decreased capillary/fibre ratio. These observations are in agreement with the comparable studies of Grimby et al ${ }^{4}$ Lotta et al ${ }^{24}$ Martin et al, ${ }^{7}$ and Round et al ${ }^{8}$

Pette and Vrbova ${ }^{2}$ have reviewed the evidence that the properties of muscle fibres in a motor unit are strongly dependent on the activity of the motoneurons. Spinal transection results in reduced activity of muscle below the level of the lesions and the paraplegic subjects studied showed an increase in the proportion of type $2 \mathrm{~A}$ and $2 \mathrm{~B}$ fibres in the tibialis anterior muscle. In addition, there was an increase in the proportion of type $2 \mathrm{C}$ fibres (with the exception of subject 3 in whom conversion to a type 2 profile was virtually complete), thus indicating the presence of ongoing fibre type conversion, even after a long interval following spinal injury.

In a muscle composed of mixed fibre types the predominance of one particular fibre type may be reflected in the rate of contraction and relaxation and there is some suggestion of this in human subjects. ${ }^{25} \mathrm{In}$ the present study, the increased ratio of type 2 to type 1 fibres corresponded to a faster rise time for both twitch and tetanic contraction, as discussed in the preceding paper. ${ }^{1}$ However, the differences in the percentage of type 2 fibres $(100 \%$ type 2 fibres in subject 3 and $30-40 \%$ type 2 fibres in subject 6 ) were not always correlated with the overall increased speed of contraction of the tibialis anterior muscle. The activity of myofibrillar ATPase, upon which fibre typing is often based, is therefore unreliable as an indicator of the speed of contraction in abnormal muscles.

Abnormally high atrophy factors were evident in initial biopsies of the paraplegic subjects and these probably account for the reduction in torque developed by the tibialis anterior muscle (see preceding paper. ${ }^{1}$ ). The mean fibre diameters of individual fibre types, with the exception of subject 5 (type 1 fibres), however, lay within the normal ranges described by Polgar et al, ${ }^{23}$ indicating that in abnormal human muscle an inspection of mean fibre diameters alone does not provide an assessment of the extent of atrophy. Preferential fibre type atrophy may be determined from the atrophy factors of individual fibre types. Atrophy was present in all fibre types in the present study in agreement with similar studies in human subjects, ${ }^{4,7}$ although it was more marked in type 1 fibres than in type $2 \mathrm{~A}$ or type $2 \mathrm{~B}$ fibres.

In the present study, the activity of SDH in the initial biopsies of the paraplegic subjects was low compared to that of the disease control group of subjects, and this was matched by a decrease in fatigue resistance. ${ }^{1}$ Those subjects with the lowest mean SDH activity also demonstrate a reduced capillary/fibre ratio and the greatest percentage of type $2 \mathrm{~B}$ fibres (subjects 3,4 and $5)$. Furthermore, muscles with the greatest percentage of type $2 \mathrm{~B}$ fibres showed the least resistance to fatigue. Thus the properties of the different fibre types appear to remain appropriately matched following spinal cord injury.

The pattern of change of muscle fibre sizes was not related to the period of time following spinal transection and the first biopsy, 1-14 years in the present study. The relationship of the degree of fibre type conversion to the period following transection, in the study of soleus and gastrocnemius by Lotta $e t a^{24}$ in subjects with cervical and upper thoracic transections, was not observed. Neurotrophic factors ${ }^{26}$ and the presence of muscle spasms may act to preserve fibre diameters ${ }^{4,5}$ in some paraplegic subjects to a greater extent than in others.

\section{Changes following a 4 week period of electrical stimulation}

Following the period of electrical stimulation there was a significant increase in the activity of $\mathrm{SDH}$, but no clear trends were observed in fibre type, fibre diameter or capillary supply. In agreement with Martin et $\mathrm{l}^{7}$ the period of training with electrical stimulation had no clear effect on the incidence of muscle fibre atrophy which was matched by the lack of any significant increase in the ability of tibialis anterior to generate more torque following stimulation. ${ }^{1}$

There was no clear evidence in the present study of a pattern of change in the proportions of fibre types following electrical stimulation. In contrast, Munsat et $a l^{27}$ observed an increase in the percentage of type 1 fibres following an electrical stimulus regime; however, the stimulation was applied for 12 weeks representing a longer and more intensive period of training. It is well known that changes in myosin isoforms from fast to slow in response to electrical stimulation take place at a later stage than changes in the various other proteins associated with speed of contraction, for example, the 
$\mathrm{Ca}^{++}$pumping ATPase system of the sarcoplasmic reticulum..$^{28}$ The stimulus regimes used in the present study may have elicited changes in the latter system, while being of insufficient duration to affect the myosin isoform transitions which would be reflected in the myofibrillar ATPase activity, demonstrable histochemically. The slowing of the rate of tetanic contraction and relaxation measured in the previous paper ${ }^{1}$ may have been explained by a change in the properties of the sarcoplasmic reticulum, which in the rabbit have been observed to occur in parallel with changes in contraction time and precede a change in the fibre type..$^{29}$

Improved resistance to fatigue is associated with increased oxidative capacity of the muscle and an increase in capillary supply. The level of SDH activity correlates with fatigue resistance.$^{30}$ In the present study a significant increase in the activity of SDH occurred in the stimulated muscles of all subjects, although the extent of SDH increase did not always correlate with the degree of increase in fatigue resistance. Subject 4 demonstrated the largest increase in $\mathrm{SDH}$, which, however, was accompanied by a decrease in fatigue resistance, which may be explained by marked muscle damage.

An increase in capillary density, associated with increased oxidative metabolism, often precedes an increase in SDH activity. ${ }^{31}$ With the exception of subject 4 in the present study, there was no clear evidence for greater capillary density or capillary/fibre ratio following the period of electrical stimulation. An improvement in capillary supply has been observed in human subjects following endurance training ${ }^{32}$ and electrical stimulation. ${ }^{33}$ However, in these situations stimulation resulted in an increase above the normal level of muscle activity. In contrast, stimulation in the present study for $5 \%$ of daily time probably represents only the normal amount of activity for the tibialis anterior muscle and may not have been sufficient to stimulate an increase in capillary supply within a period of 4 weeks.

Delitto and Snyder-Mackler ${ }^{34}$ have suggested that stimulation of muscle with surface electrodes, selectively recruits and trains type 2 fibres, as a result of an inverted recruitment order. However, the increase of $\mathrm{SDH}$ activity in the present study occurred in all fibre types, suggesting a sample of all muscle fibres was recruited.

The subsarcolemmal vacuolation seen in five of the seven biopsies in the stimulated limbs was an unexpected finding. Such vacuolation is not common in muscle histopathology, being largely confined to clinical conditions of glycogen storage, inflammatory myopathy or intoxication with substances such as chloroquine. The significance of the finding in the present study is unclear, but initial fears that it might indicate severe damage to the muscle fibre membrane were not substantiated, since serum creatine kinase (CK) levels were not raised in the subjects, where this was measured (subjects 6 and 7), despite the presence of vacuolation. Creatine kinase has been effectively used to assess muscle damage following stepping exercise in human subjects. ${ }^{35}$ However, it would appear that CK estimation may not be sufficiently sensitive to monitor muscle damage related to a regime of electrical stimulation.

\section{Conclusion}

In this and the preceding paper ${ }^{1}$ marked changes of the contractile properties in the tibialis anterior muscle have been observed in subjects following complete thoracic spinal cord transection. Despite prolonged disuse, it is encouraging that resistance to fatigue and speed of contraction, together with oxidative capacity, rapidly revert towards normal values in response to daily treatments with electrical stimulation at only twice motor threshold. The period of time following spinal injury does not appear to influence adversely the retraining of muscle properties by electrical stimulation. In subjects with other central nervous or orthopaedic pathological conditions eg partial spinal transection, hemiplegia, head injury, cerebral palsy, orthopaedic trauma, the method is also likely to be of value in preventing the changes associated with disuse or in retraining contractile properties. In fact, electrical stimulation up to twice motor threshold for a daily period comparable to that used in the training of the paraplegic subjects was found to be tolerable by one of the authors, whose somatic sensation was intact. The effects of training on force and fibre type profile in paraplegic subjects, however, remain open and require further study. It is likely that the period of electrical stimulation needs to be longer than 4 weeks and that the muscle stimulated should contract under load, if these parameters are to be significantly altered.

\section{Acknowledgements}

We acknowledge with gratitude financial support from the Northern Regional Health Authority, Dame Catherine Cookson and the Regional Spinal Injuries Unit Consultants' Trust Fund, Hexham General Hospital. We would like to thank Professor Daniel Kernell for helpful discussions in the initiation of the project.

\section{References}

1 Rochester $\mathrm{L}$ et al. Influence of electrical stimulation on the tibialis anterior muscle in paraplegic subjects. 1. Contractile properties. Paraplegia 1995; 33: 510-513.

2 Pette D, Vrbova G. Neural control of phenotypic expression in mammalian muscle fibres. Muscle Nerve 1985; 8: 676-689.

3 Navarrete R, Vrbova G. Activity-dependent interactions between motoneurons and muscles: their role in the development of the motor unit. Prog Neurobiol 1993; 41: 93-124.

4 Grimby L, Broberg C, Krotkiewska T, Krotkiewski M. Muscle fibre composition in patients with traumatic cord lesions. Scand J Rehabil Med 1976; 8: 37-42.

5 Edström L. Selective changes in the size of red and white muscle fibres in upper motor lesions and parkinsonism. $J$ Neurol Sci 1970; 11: 537-550.

6 Edström L. Selective atrophy of red muscle fibres in the quadriceps in long-standing knee-joint dysfunction injuries to the anterior cruciate ligament. J Neurol Sci 1970; 11: 551-558.

7 Martin TP, Stein RB, Hoeppner PH, Reid DC. Influence of electrical stimulation on the morphological and metabolic properties of paralysed muscle. J Appl Physiol 1992; 72: 1401-1406. 
8 Round JM, Barr FMD, Moffat B, Jones DA. Fibre areas and histochemical fibre types in the quadriceps muscle of paraplegic subjects. J Neurol Sci 1993; 116: 207-211.

9 Gibson JNA, Smith K, Rennie MJ. Prevention of disuse muscle atrophy by means of electrical stimulation: maintenance of protein synthesis. Lancet 1988 Oct: 767-770.

10 Cooper RG, Stokes MJ, Gibson H, Edwards RHT. Minimising of fatigue for functional electrical stimulation of muscle. Clin Rehabil 1989; 3: 333-340.

11 Stein RB et al. Optimal stimulation of paralysed muscle after human spinal cord injury. J Appl Physiol 1992; 72: 1393-1400.

12 Kernell D, Donselaar Y, Eerbeek O. Effects of high and low-rate chronic stimulation on cat's fast muscle. I. Speed and force-related properties. J Neurophysiol 1987; 58: 598-613.

13 Hendriksson $\mathbf{J}$ et al . Chronic stimulation of mammalian muscle: changes in enzymes of six metabolic pathways. Am J Physiol 1986; 251 : 614-632.

14 Kugelberg E, Lindegren B. Transmission and contraction fatigue of rat motor units in relation to succinate dehydrogenase activity of motor unit fibres. J Physiol (Lond) 1979; 288: 285-300.

15 Rochester L et al. Influence of electrical stimulation on contractile and histochemical properties of tibialis anterior muscle in paraplegic subjects. J Physiol (Lond) 1992; 45: 278P.

16 Brooke $\mathrm{MH}$, Kaiser KK. Muscle fiber types: how many and what kind? Arch Neurol 1970; 23: 369-379.

17 Song SK, Shimada N, Anderson PJ. Orthogonal diameters in the analysis of muscle fibre size and form. Nature 1963; 200: $1220-1221$.

18 Brooke $\mathrm{MH}$, Engel WK. The histographic analysis of human muscle biopsies with regard to fiber types. II. Diseases of the upper and lower motor neurons. Neurology 1969; 19: 378-393.

19 Old SL, Johnson MA. Methods of microphotometric assay of succinate dehydrogenase and cytochrome c oxidase activities for use on human skeletal muscle. Histochem J 1989; 21: 545-556.

20 Holthofer $\mathrm{J}$ et al. Ulex europeaus I lectin as a marker for vascular endothelium in human tissues. Lab Invest 1982; 47: 60-66.

21 Johnson MA, Polgar J, Weightman D, Appleton D. Data on the distribution of fibre types in thirty-six human muscles. An autopsy study. J Neurol Sci 1973; 18: 111-129.

22 Dubowitz V, Brooke MH. Muscle Biopsy: A Modern Ap- proach. 1st edn. WB Saunders: London, Philadelphia \& Toronto, 1973.

23 Polgar J, Johson MA, Weightman D, Appleton D. Data on fibre size in thiry-six human muscles. An autopsy study. J Neurol Sci 1973; 19: 307-318.

24 Lotta et al. Morphometric and neurophysiological analysis of skeletal muscle in paraplegic patients with traumatic cord lesion. Paraplegia 1991; 29: 247-252.

25 McComas AJ, Thomas HC. Fast and slow twitch muscles in man. J Neurol Sci 1968; 7: 301-307.

26 Buller AJ, Eccles JC, Eccles RM. Differentiation of fast and slow muscles of the cat hind limb. J Physiol (Lond) 1960; 150: 399-416.

27 Munsat TL, McNeal D, Waters R. Effects of nerve stimulation on human muscle. Arch Neurol 1976; 33: 608-617.

28 Jolesz F, Sreter FA. Development, innervation and activity pattern induced changes in skeletal muscle. Annu Rev Physiol 1981; 43: 531-552.

29 Heilman C, Pette D. Molecular transformation in sarcoplasmic reticulum of fast-twitch muscle by electrostimulation. Eur $J$ Biochem 1979; 93: 437-446.

30 Burke RE et al. Mammalian motor units: physiological-histochemical correlation in three types in cat gastrocnemius. Science 1971; 174: 709-712.

31 Brown MD, Cotter MA, Hudlicka O, Vrbova G. The effects of different patterns of muscle activity on capillary density, mechanical properties and structure of slow and fast rabbit muscles. Pflügers Arch 1976; 361: 241-250.

32 Saltin B, Gollnick PD. Skeletal muscle adaptability: significance for metabolism and performance. In: Peachy LD, Adrian RH, Geiger SR, (eds). Handbook of Physiology, Section 10, Skeletal muscle. American Physiology Society: Bethesda, 1983, pp 555-631.

33 Cabric M, Appel HJ, Resic A. Stereological analysis of capillaries in electrostimulated human muscles. Int J Sports Med 1987; 8: 327-330.

34 Delitto A, Synder-Mackler L. Two theories of strength augmentation using percutaneous electrical stimulation. Phys Ther 1990; 70: 158-164.

35 Newham DJ, Jones DA, Edwards RHT. Large delayed plasma creatine kinase changes after stepping exercise. Muscle Nerve 1983; 6: 380-385. 